\title{
Morphologic and Functional Changes in Nontumorous Liver Tissue After Radiofrequency Ablation in an In Vivo Model: Comparison of ${ }^{18}$ F-FDG PET/CT, MRI, Ultrasound, and CT
}

\author{
Florian M. Vogt ${ }^{1}$, Gerald Antoch ${ }^{1}$, Patrick Veit ${ }^{1}$, Lutz S. Freudenberg ${ }^{2}$, Nina Blechschmid ${ }^{1}$, Olaf Diersch ${ }^{3}$, \\ Andreas Bockisch ${ }^{2}$, Jörg Barkhausen ${ }^{1}$, and Hilmar Kuehl ${ }^{1}$ \\ ${ }^{1}$ Department of Diagnostic and Interventional Radiology and Neuroradiology, University Duisburg-Essen, Essen, Germany; \\ ${ }^{2}$ Department of Nuclear Medicine, University Duisburg-Essen, Essen, Germany; and ${ }^{3}$ Department of Pathology, \\ University Cologne, Cologne, Germany
}

Rimlike contrast enhancement on morphologic imaging and increased tracer uptake on ${ }^{18} \mathrm{~F}$-FDG PET in the periphery of the necrosis can hamper differentiation of residual tumor from regenerative tissue after radiofrequency ablation of liver lesions. This study used MRI, CT, ultrasound, and ${ }^{18} \mathrm{~F}-\mathrm{FDG}$ PET/CT to assess the typical appearance of lesions in nontumorous animal liver tissue after radiofrequency ablation. Methods: Lesions were created by radiofrequency ablation of normal liver parenchyma in 21 minipigs. Follow-up was performed by 3 contrast-enhanced morphologic modalities-MRI, CT, and ultrasound-and by ${ }^{18} \mathrm{~F}-$ FDG PET/CT immediately, 3 and $10 \mathrm{~d}$, and 1, 2, 3, and 6 mo after radiofrequency ablation. Images were evaluated qualitatively for areas of increased enhancement and regions of elevated tracer uptake. Furthermore, all images were assessed quantitatively by determination of ratios comparing enhancement/tracer uptake in the periphery of the necrosis with enhancement/tracer uptake in normal liver parenchyma. Imaging findings were compared with histopathology findings. Results: Immediately after radiofrequency ablation, no increase in ${ }^{18} \mathrm{~F}$ FDG uptake was visible, whereas elevated enhancement was noticed in the periphery of the necrosis on all morphologic imaging procedures. At further follow-up, an area of rimlike increase in ${ }^{18} \mathrm{~F}-\mathrm{FDG}$ uptake surrounding the necrosis was detected on $\mathrm{PET} / \mathrm{CT}$. The rimlike pattern of increased enhancement in the arterial phase was present for all liver lesions on CT, MRI, and ultrasound, especially between day 3 and month 1 after the radiofrequency ablation. Both elevated glucose metabolism and enhancement persisted for 6 mo postinterventionally. Histologic examination showed a hemorrhagic border converting into a regeneration capsule. Conclusion: If performed immediately after radiofrequency ablation, ${ }^{18} \mathrm{~F}-\mathrm{FDG} \mathrm{PET} / \mathrm{CT}$ probably has benefits over those of morphologic imaging procedures when assessing liver tissue for residual tumor. Later follow-up may be hampered

Received Apr. 18, 2007; revision accepted Jul. 31, 2007.

For correspondence or reprints contact: Florian M. Vogt, MD, Department of Diagnostic and Interventional Radiology and Neuroradiology, University of Duisburg-Essen, Hufelandstrasse 55, D-45122 Essen, Germany.

E-mail: florian.vogt@uni-essen.de

COPYRIGHT @ 2007 by the Society of Nuclear Medicine, Inc. by visualization of peripheral hyperperfusion and tissue regeneration. Further studies on a patient population are essential.

Key Words: minimally invasive technique; tissue necrosis; periablational region; peripheral enhancement; tissue response

J Nucl Med 2007; 48:1836-1844

DOI: 10.2967/jnumed.107.042846

$\mathbf{I}$ patients with liver malignancies, surgical resection has been considered the curative treatment of choice. Despite surgical advances resulting in an improved outcome, with 5 -y survival rates of up to $50 \%$ in a selected cohort (1), only a minority of patients have been eligible for hepatic resection (2). Minimally invasive techniques such as radiofrequency ablation, laser-induced interstitial thermotherapy, and microwave ablation have emerged as attractive alternatives to provide local destruction of hepatic tumors. With the introduction of modified electrodes $(3,4)$, radiofrequency ablation must be considered the most widely accepted technique for interventional treatment of malignant liver tumors in patients not eligible for surgical therapy $(1,5)$. In radiofrequency ablation, a high-frequency alternating current $(350-500 \mathrm{kHz})$ is used to generate frictional heat energy causing thermal coagulative necrosis. The success of ablative treatment strongly depends on complete destruction of the tumor, along with safety margins. However, residual tumor cells or local tumor recurrence in tissue adjacent to the ablated site frequently has been reported $(1,6-9)$ and has been attributed to incomplete ablation of tumor cells during the treatment $(10)$. With respect to postprocedural imaging follow-up, it is critical to detect residual or recurrent tumor as early as possible. On the other hand, false-positive diagnoses of residual disease leading to unnecessary biopsies or treatment need to be minimized. The term irregular peripheral enhancement, recommended 
by the International Working Group on Image-Guided Tumor Ablation, has been used to describe residual or recurrent tumor at the treatment margin as seen on enhanced images. This term needs to be differentiated from the term benign periablational enhancement (11), which describes a transient enhanced-imaging finding that frequently occurs immediately after ablation and may be visible for up to 6 mo afterward. This finding represents a benign physiologic response to thermal injury and usually manifests as a thin, rimlike peripheral zone around the ablation necrosis (10). Despite extensive research on the diagnostic performance of ultrasound, CT, and MRI for the localization of residual tumor cells, and despite recent and ongoing developments in contrast agents, differentiation between benign periablational enhancement and small residual or recurrent tumor remains challenging on follow-up examinations $(9,12,13)$. Even the application of ${ }^{18} \mathrm{~F}$-FDG PET does not allow reliable differentiation of local tissue regeneration from residual tumor, because both can go along with increased glucose metabolism (14-16).

Therefore, understanding tissue response to radiofrequency electrocautery immediately after the procedure and during long-term follow-up is important. In vivo radiologicpathologic correlations for radiofrequency tumor ablation have been rare and have encompassed only short-term follow-up periods $(9,10,17,18)$. This study was performed to assess the typical appearance of nontumorous liver tissue in an animal model up to 6 mo after radiofrequency ablation on 3 contrast-enhanced morphologic modalitiesdynamic MRI, ultrasound, and CT-and on ${ }^{18}$ F-FDG PET and ${ }^{18}$ F-FDG PET/CT. Radiofrequency ablation was performed on nontumorous liver tissue in 21 Göttingen minipigs (Ellegaard), and the imaging findings were correlated with histopathologic findings. Preliminary short-term follow-up results from this study have been published (19). A rimlike increase in enhancement was found on morphologic imaging immediately after radiofrequency ablation, whereas homogeneous tracer distribution surrounding the area of necrosis could be detected on functional imaging.

\section{MATERIALS AND METHODS}

\section{Animal Model}

The study was conducted on 21 male Göttingen minipigs (initial weight, $30-45 \mathrm{~kg}$ ), who underwent 2 applications of radiofrequency ablation to the right and left liver lobes during the same session. The animals were kept in sheltered runs at the central animal facility and received a standard pig meal ad libitum. Before the intervention and the subsequent imaging procedures, all animals were kept fasting for a minimum of $8 \mathrm{~h}$. Blood glucose, common hematology values, urea and electrolytes, white blood count, and C-reactive protein were measured before the intervention and before all follow-up imaging examinations. Intramuscular injections of $50 \mathrm{mg}$ of ketamine hydrochloride (Ketamine 10\%; Ceva Tiergesundheit $\mathrm{GmbH}$ ) per kilogram of body weight and $0.12 \mathrm{mg}$ of atropine were administered to each pig for induction of anesthesia. For the intervention and imaging procedures, the animals were sedated by an injection of midazolam hydrochloride
(Dormicum; Hoffmann-La Roche AG) via an intravenous cannula placed in an ear vein. Analgesia was administered intermittently using fentanyldihydrogencitrate (Fentanyl Hexal; Hexal AG) and midazolam hydrochloride. Cardiac and respiratory parameters were monitored throughout the experiment. The animal study was approved by the supervising state agency (license number G680/02) and was performed in full accordance with all state and federal guidelines.

\section{Radiofrequency Ablation}

After a sterile field had been prepared, radiofrequency ablation was performed under CT guidance with the Cool-tip system (Radionics), comprising a dual-lumen, internally cooled electrode to prevent tissue charring (20). The electrode is cooled internally by continuous perfusion of the lumen with cooled saline. A singleneedle electrode (17-gauge) with $3-\mathrm{cm}$ exposure of the distal tip was chosen for all interventions. The probe length was either 10 or $15 \mathrm{~cm}$ based on the individual anatomic situation. Two grounding pads were placed on the shaved abdominal wall of each pig. The radiofrequency current was generated by a commercially available 200-W power supply (Radionics). Radiofrequency ablation was performed for $20 \mathrm{~min}$ per lesion, with the generator continuously monitoring tissue impedance and adjusting power output. Ablation was performed on the right and the left liver lobes. On termination of each ablation, the saline perfusion was stopped to increase the needle-tip temperature to over $60^{\circ} \mathrm{C}$. Finally, the needle was retracted slowly to cauterize the needle track.

\section{Imaging Procedures}

All imaging procedures were performed immediately (within $120 \mathrm{~min}$ ) after radiofrequency ablation; 3 and $10 \mathrm{~d}$ after radiofrequency ablation; and 1, 2, 3, and 6 mo after radiofrequency ablation. The various procedures were performed in random order. The animals that were being included in the longer follow-up period underwent all the examinations planned immediately and at the various intervals until the planned sacrifice date.

Imaging findings were compared with histopathologic findings; for that purpose, 3 minipigs were sacrificed after each follow-up imaging procedure.

\section{MRI}

MR images were acquired on a 1.5-T Sonata System (Siemens Medical Solutions). All pigs were placed supine on the examination table, using the field of view defined for the liver.

First, unenhanced images were acquired with respiratory gating using T1-weighted (repetition time/echo time, 124/1.8 ms) and T2-weighted (repetition time/echo time, 1,200/60 ms) sequences with a section width of $7 \mathrm{~mm}$. Respiratory gating was achieved using a respiratory belt, which was placed around the lower abdomen. Subsequently, a paramagnetic contrast agent (Multihance; Bracco) was administered intravenously at $2 \mathrm{~mL} / \mathrm{s}$ at a dose of 0.2 $\mathrm{mmol} / \mathrm{kg}$. Four 3-dimensional datasets (repetition time/echo time, 2.6/1.0 ms; flip angle, $25^{\circ}$; acquisition time, $16 \mathrm{~s}$ for each dataset) of the liver were acquired without enhancement and subsequently in the arterial (25-s delay), portal venous (51-s delay), and venous (78-s delay) phases. The 4 datasets were acquired during free breathing, whereas sedation was deepened for the short-term acquisition. Only mild blurring was seen in some datasets, and it did not hamper analysis. For quantitative measurement, the dataset obtained in the arterial phase was used. Finally, T1-weighted images 
with a section width of $7 \mathrm{~mm}$ were acquired during the late venous phase, 40 min after injection of the contrast agent.

\section{CT}

CT images (Biograph; Siemens Medical Solutions) of the liver were acquired with the pig supine, using $130 \mathrm{mAs}, 130 \mathrm{kV}$, a slice width of $5 \mathrm{~mm}$, and a table feed of $8 \mathrm{~mm}$ per gantry rotation. Images were reconstructed at 2.4- $\mathrm{mm}$ increments. CT was performed without enhancement and in the arterial (25-s delay) and venous (70-s delay) phases after administration of $70 \mathrm{~mL}$ of a iodinated contrast agent (flow rate, $3 \mathrm{~mL} / \mathrm{s}$ ) (Xenetix, $300[300 \mathrm{mg}$ iodine $/ \mathrm{mL}$; ; Guerbet $\mathrm{GmbH}$ ) using an automated injector (LiebelFlarsheim). Like the MRI datasets, images acquired in the arterial phase were evaluated quantitatively.

\section{Ultrasound}

Ultrasound was performed with the pig supine on a Sequoia system (Acuson) with a curved-array 2.5- to 5- $\mathrm{MHz}$ transducer. Imaging was performed before and after intravenous bolus injection of $2.5 \mathrm{~mL}$ of a microbubble-based ultrasound contrast agent (Sonovue; Bracco International) per lesion. All images of the 2 lesions were acquired with and without enhancement in the arterial and portal venous phases. Enhanced images were acquired using a low mechanical index (0.2). The images were stored digitally and on videotape.

\section{PET/CT}

Combined PET/CT was performed on a Biograph scanner (Siemens Medical Solutions), which comprises a dual-slice spiral CT component and a full-ring PET component. The PET component has an in-plane spatial resolution of $4.6 \mathrm{~mm}$ and an axial field of view of $15.5 \mathrm{~cm}$ for 1 table position. CT and PET datasets can be viewed separately or after image fusion on a computer workstation.

PET images were acquired with the pig supine $60 \mathrm{~min}$ after the administration of $250 \mathrm{MBq}$ of ${ }^{18} \mathrm{~F}-\mathrm{FDG}$, and the same field of view was covered as for CT. Blood glucose levels were confirmed to be within the normal range before injection of the radioactive tracer. The time to acquire a single bed position was set at $7 \mathrm{~min}$. PET images were corrected for attenuation and scatter on the basis of the CT data (21). The images were iteratively reconstructed (2 iterations and 8 subsets).

\section{Image Evaluation and Data Analysis}

Ultrasound images, MR images, and CT images were each assessed by 2 radiologists, whereas PET and fused PET/CT images were read by a radiologist in conjunction with a nuclear medicine physician. For qualitative analysis, all data were evaluated as 1 pool of images with regard to the different phases of the experiment. Images were assessed qualitatively for areas of altered enhancement on morphologic imaging procedures and for regions of altered glucose use on functional imaging. Images were qualitatively assessed by comparing arterial enhancement (morphology) and tracer uptake (PET and PET/CT) in the periphery of the ablative necrosis with the untreated liver parenchyma, and potential differences were rated according to a 3-score scale. A score of 1 indicated no enhancement or glucose metabolism in the region surrounding the necrosis (lesion periphery), compared with untreated liver parenchyma. A score of 2 indicated a mild increase in enhancement or glucose use surrounding the ablative necrosis, compared with untreated liver tissue. A score of 3 indicated a strong increase in enhancement or glucose use in the tissue surrounding the necrosis, compared with untreated liver parenchyma.

In addition, all images were assessed by the same radiologists quantitatively on a workstation. The lesion size was assessed on axial images on the basis of measurements of both the small-axis and the large-axis diameters, including the transient zone, according to the recommendations of the International Working Group on Image-Guided Tumor Ablation and was compared with the standard of reference (11). Lesion size on PET/CT was assessed in the fused mode. Because variations in the threshold can create significant changes in the size of the lesion, the PET threshold was adjusted to $50 \%$ of the maximum standardized uptake value for this purpose. The effect of radiofrequency ablation on enhancement was assessed by determining 2 ratios -1 for arterial-phase MRI and 1 for CT-based on measurements of signal intensity in regions of interest each containing at least 30 pixels. Ratio 1, an enhancement ratio between the center of ablative necrosis and normal liver parenchyma, was calculated as

$$
\mathrm{r}_{\mathrm{c} / \mathrm{p}}=\frac{\text { enhancement, lesion center }}{\text { enhancement, normal parenchyma }}
$$

Ratio 2, an enhancement ratio between the periphery of ablative necrosis and normal liver parenchyma, was calculated as

$$
r_{p / p}=\frac{\text { enhancement, lesion periphery }}{\text { enhancement, normal parenchyma }}
$$

Enhancement was quantified in Hounsfield units for CT and signal intensity for MRI. Software for quantification of enhancement on ultrasound images is not yet commercially available.

The effect of treatment on PET tracer quantification near the necrosis and in its periphery was assessed by determining 2 ratios like those used for the other imaging procedures (regions of interest each containing at least 30 pixels; mean values). Ratio 1, a tracer activity ratio between the center of ablative necrosis and normal liver parenchyma, was calculated as

$$
\mathrm{r}_{\mathrm{c} / \mathrm{p}}=\frac{\text { activity concentration, lesion center }}{\text { activity concentration, normal parenchyma }} \text {. }
$$

Ratio 2, a tracer activity ratio between the periphery of ablative necrosis and normal liver parenchyma, was calculated as

$$
\mathrm{r}_{\mathrm{p} / \mathrm{p}}=\frac{\text { activity concentration, lesion periphery }}{\text { activity concentration, normal parenchyma }} .
$$

\section{Histopathology}

After radiofrequency ablation and subsequent imaging, 3 animals were sacrificed for histopathologic evaluation of the ablative necroses at each time point. The same pathologist assessed all tissue samples for areas of increased tissue regeneration.

Liver tissue samples from the ablated regions and the untreated liver parenchyma were fixed in $4 \%$ buffered formalin and embedded in paraffin. Three-micrometer sections were stained with hematoxylin-eosin, periodic acid-Schiff, and Elastica-van Gieson.

The lesions were photodocumented using a microscope (Eclips 80i; Nikon) equipped with a digital camera (DXM 1200F; Nikon). Single pictures were stitched to slide strips using a software 
program (Panavue; Panavue Inc.). The slides were evaluated by a pathologist for areas of necrosis, regeneration, scar formation, and inflammatory reaction.

\section{RESULTS}

\section{Radiofrequency Ablation}

Blood glucose levels and the blood samples were found to be normal before the intervention and before all imaging procedures for all animals. Radiofrequency ablation was performed without complications in all 21 pigs. In 2 pigs, only 1 radiofrequency ablation could be performed because of a hypoplastic left hepatic lobe. One of the pigs was assigned to a group that was sacrificed 1 mo after radiofrequency ablation, and the other was sacrificed 2 mo after the interventional procedure. Therefore, data analysis was based on 40 lesions in 21 pigs.

\section{Histopathology}

Three different zones were detected on histopathologic evaluation of the ablative site immediately after radiofrequency ablation. A central zone demonstrated early signs of tissue necrosis and a marked dissociation of hepatocytes. A transitional zone showed sinusoids engorged with blood because the central ablative necrosis was obstructing outflow. A peripheral zone demonstrated mild reactive changes, that is, vacuole transformation of cytoplasm and increased sinusoidal perfusion.

After 3-10 d, coagulation necrosis of the liver tissue was observed in the center of the lesion. An area of necrotic liver tissue with focal granulocytic infiltration and the beginnings of granulation tissue formation was found separating the viable liver tissue from the central necrosis. In this area, sinusoids showed focal dilatation and accumulation of blood. Liver cells showed signs of regeneration, including single mitotic figures. Between $10 \mathrm{~d}$ and 6 mo after radiofrequency ablation, regeneration transformed gradually to scar tissue.

After 6 mo, normal liver tissue surrounded a wide rim of scar tissue that encircled central confluent necrotic tissue. The scar was more cellular toward the normal liver tissue, including small capillaries and a minor infiltrate of lymphocytes and single plasma cells. Toward the central necrosis of the lesion, hypocellularity and focal calcifications were observed in the scar tissue (Figs. 1 and 2).

\section{Mean Lesion Size}

According to the measurement of tissue samples, the mean lesion size was $3.9 \mathrm{~cm}$ in the large-axis diameter and $2.3 \mathrm{~cm}$ in the small-axis diameter and decreased to 2.0 and $1.6 \mathrm{~cm}$, respectively, after $6 \mathrm{mo}$. The standard of reference and all imaging modalities showed a comparable decrease in lesion size. The average decrease within 6 mo was $48 \%$ in the longitudinal diameter and $30 \%$ in the transverse diameter. Ultrasound, CT, MRI, and PET/CT determined both diameters equally well, compared with the standard of reference, except for the last 3 follow-up examinations (2- to 6-mo control), at which all morphologic imaging modalities slightly overestimated the lesion size. The mean largeaxis and small-axis diameters of radiofrequency-induced lesions as determined with the different imaging procedures are summarized in Table 1.

\section{Qualitative Image Analysis}

On CT, MRI, and ultrasound, the center of the ablative coagulation was characterized as hypodense, hypointense, or hypoechoic, respectively, compared with normal liver parenchyma. After administration of the contrast agent, a marked enhancement was identified surrounding all ablative necroses on ultrasound images, CT images, and MR images. This area of enhancement corresponded to the transient zone and, later, to the area of surrounding fibrosis and was detected on all follow-up examinations. The mean qualitative scores for this rim of enhancement were $2.4 \pm$ 0.5 for MRI, $2.7 \pm 0.5$ for $\mathrm{CT}$, and $2.5 \pm 0.5$ for ultrasound immediately after radiofrequency ablation. On further followup at $6 \mathrm{mo}$, the rimlike enhancement decreased to $2.0 \pm$ $0.5,1.9 \pm 0.4$, and $2.3 \pm 0.4$, respectively. When PET images were assessed qualitatively immediately after the ablation procedure, the area of coagulation was characterized by decreased glucose metabolism. Homogeneous tracer use was found in the livers of all ablated pigs when liver tissue surrounding the coagulation necrosis was compared with normal liver parenchyma. There were no areas of a focal or rimlike increase in glucose metabolism adjacent to the ablative necrosis. Qualitative analysis revealed a mean score of $1 \pm 0$ (Fig. 3). However, an increase in tracer use in the ablative margin surrounding the coagulation necrosis was detected in all animals at the 3-d follow-up. This increase in glucose uptake was visible up to 6 mo after radiofrequency ablation (Fig. 4). Although a mean qualitative score of $2.2 \pm 0.4$ was calculated for PET images acquired $3 \mathrm{~d}$ after the interventional procedure, the score decreased to a mean of $1.4 \pm 0.5$ after 6 mo.

\section{Quantitative Image Analysis}

The mean $r_{c / p}$ immediately after the interventional procedure was $0.70 \pm 0.11$ for $\mathrm{CT}, 0.51 \pm 0.17$ for MRI, and $0.63 \pm 0.10$ for PET/CT. For CT, the ratio slightly decreased between days 1 and 3 and stayed relatively constant thereafter (range, 0.59-0.65), whereas the ratio for MRI continuously decreased over time. For PET, mean ratios increased continuously within the first month, whereas the following examinations were characterized by a decrease in ratios.

Corresponding to the qualitative image findings, increased enhancement could be found in the periphery after radiofrequency ablation (Fig. 4). The $r_{p / p}$ ratio amounted to $1.52 \pm$ 0.21 for CT immediately after radiofrequency ablation and peaked at day $3(1.57 \pm 0.20)$, whereas an $\mathrm{r}_{\mathrm{p} / \mathrm{p}}$ ratio of $1.55 \pm$ 0.23 was found for MRI, with the peak mean ratio occurring on day $10(1.85 \pm 0.58)$. A slow decrease in the enhancement ratios was seen on both imaging modalities (Fig. 5).

The mean diameter of the rim of enhancement measured $4.8 \pm 2.1 \mathrm{~mm}$ on CT images, $3.0 \pm 0.8 \mathrm{~mm}$ on MR images, 

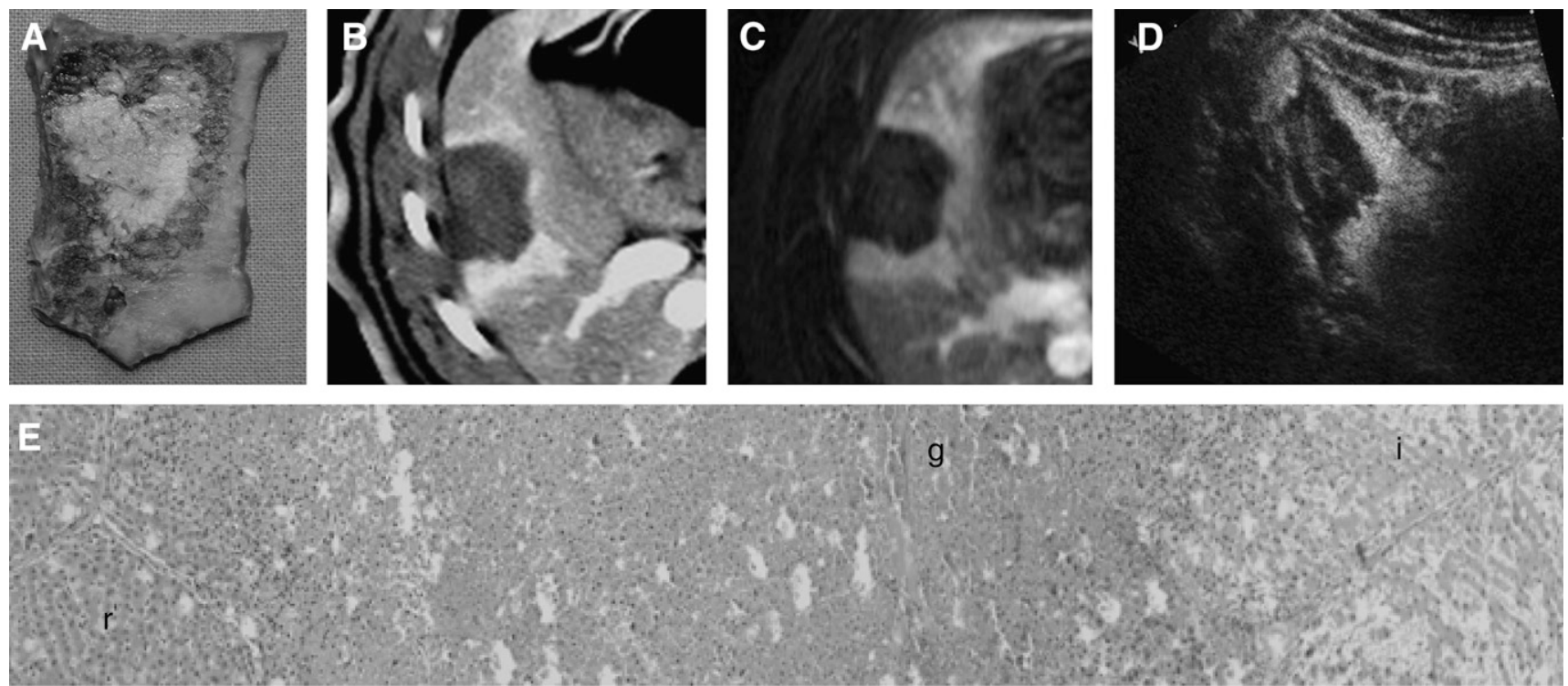

FIGURE 1. Macroscopic tissue sample (A) obtained $3 \mathrm{~d}$ after radiofrequency ablation further shows blood-filled sinusoids and extent of reactive changes in periphery of necrosis, compared with tissue samples sectioned immediately after radiofrequency ablation. Morphologic imaging (CT [B], MRI [C], and ultrasound [D]) depict pronounced and extended enhancement in periphery of necrosis. Enhancement corresponded on histopathology images (E) to mixture of engorged sinusoids and beginning inflammatory reaction, with granulation and hyperemia. Regenerating liver tissue $(r)$ surrounds zone of necrotic liver tissue, with focal granulocytic infiltrate $(\mathrm{g})$ and inner zone of necrosis (i).

and $2.8 \pm 1.6 \mathrm{~mm}$ on ultrasound images immediately after radiofrequency ablation (Fig. 6). Although 3-10 d after radiofrequency ablation the rim enhancement along the ablated tissue became more prominent than that immediately after radiofrequency ablation and often was irregular in shape, the extent of the arterial enhancement consequently decreased over time.

Quantitative evaluation of the lesion periphery, compared with normal liver tissue, on PET demonstrated homogeneous glucose use immediately after the interventional procedure $\left(\mathrm{r}_{\mathrm{p} / \mathrm{p}}\right.$ ratio, $\left.1.01 \pm 0.05\right)$. Increased glucose use in the lesion periphery, with a peak mean ratio of $1.19 \pm$ 0.09 , was found between day 3 and month 1 after radiofrequency ablation, followed by a decrease back to almost homogeneous glucose use after 6 mo (1.07 \pm 0.09) (Fig. 4).

\section{DISCUSSION}

Thermal injury of normal liver tissue due to radiofrequency ablation leads to changes in the periphery of the induced necrosis - changes that can be detected on histopathologic examination and with different imaging modalities.
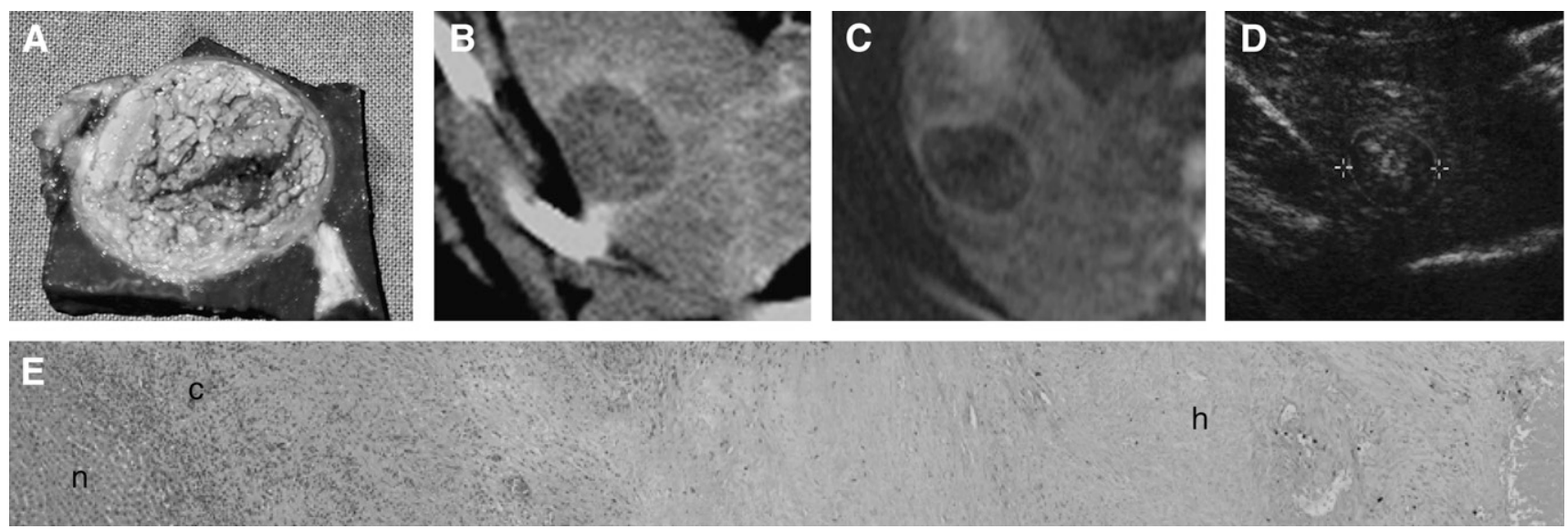

FIGURE 2. Macroscopic tissue sample (A) obtained 6 mo after radiofrequency ablation demonstrates well-defined capsule surrounding necrosis, with maximum diameter of $3 \mathrm{~mm}$, corresponding to thin-rim peripheral enhancement on morphologic images (CT [B], MRI [C], and ultrasound [D]) and fibrosis on histopathology images (E). Normal liver (n) surrounds cellular scar tissue (c) transforming into hypocellular scar tissue $(\mathrm{h})$ and central necrosis. 
TABLE 1

Morphologic Measurements of Coagulation on All Imaging Modalities, Compared with Standard of Reference

\begin{tabular}{|c|c|c|c|c|c|c|c|}
\hline Imaging modality & $3 \mathrm{~h}$ & $3 d$ & $10 \mathrm{~d}$ & $1 \mathrm{mo}$ & $2 \mathrm{mo}$ & $3 \mathrm{mo}$ & $6 \mathrm{mo}$ \\
\hline \multicolumn{8}{|l|}{ CT } \\
\hline$n$ & 40 & 34 & 28 & 22 & 17 & 12 & 6 \\
\hline Longitudinal diameter $(\mathrm{cm})$ & $4.00 \pm 0.50$ & $4.00 \pm 0.64$ & $3.54 \pm 0.56$ & $3.03 \pm 0.61$ & $2.53 \pm 0.54$ & $2.37 \pm 0.56$ & $2.16 \pm 0.83$ \\
\hline Transverse diameter $(\mathrm{cm})$ & $2.52 \pm 0.50$ & $2.53 \pm 0.64$ & $2.38 \pm 0.51$ & $1.95 \pm 0.37$ & $1.78 \pm 0.33$ & $1.76 \pm 0.50$ & $1.54 \pm 0.69$ \\
\hline \multicolumn{8}{|c|}{ 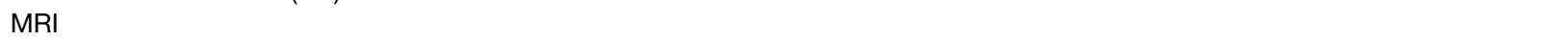 } \\
\hline$n$ & 40 & 34 & 28 & 22 & 17 & 12 & 6 \\
\hline Longitudinal diameter $(\mathrm{cm})$ & $4.15 \pm 0.63$ & $4.05 \pm 0.69$ & $3.67 \pm 0.69$ & $3.03 \pm 0.54$ & $2.67 \pm 0.52$ & $2.53 \pm 0.57$ & $2.40 \pm 0.83$ \\
\hline Transverse diameter $(\mathrm{cm})$ & $2.56 \pm 0.56$ & $2.56 \pm 0.61$ & $2.37 \pm 0.53$ & $1.93 \pm 0.39$ & $1.79 \pm 0.37$ & $1.76 \pm 0.46$ & $1.64 \pm 0.60$ \\
\hline \multicolumn{8}{|c|}{ 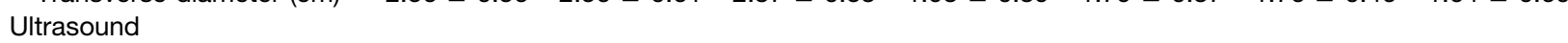 } \\
\hline$n$ & 40 & 34 & 28 & 22 & 17 & 12 & 6 \\
\hline Longitudinal diameter $(\mathrm{cm})$ & $3.80 \pm 0.69$ & $3.77 \pm 0.49$ & $3.71 \pm 0.61$ & $3.06 \pm 0.60$ & $2.66 \pm 0.62$ & $2.63 \pm 0.66$ & $2.36 \pm 1.00$ \\
\hline Transverse diameter $(\mathrm{cm})$ & $2.52 \pm 0.47$ & $2.44 \pm 0.48$ & $2.29 \pm 0.49$ & $2.00 \pm 0.39$ & $1.77 \pm 0.37$ & $1.78 \pm 0.46$ & $1.54 \pm 0.59$ \\
\hline \multicolumn{8}{|l|}{$\mathrm{PET} / \mathrm{CT}$} \\
\hline$n$ & 40 & 34 & 28 & 22 & 17 & 12 & 6 \\
\hline Longitudinal diameter $(\mathrm{cm})$ & $3.97 \pm 0.82$ & $3.94 \pm 0.76$ & $3.45 \pm 0.86$ & $3.09 \pm 0.60$ & $2.65 \pm 0.46$ & $2.57 \pm 0.58$ & $2.44 \pm 0.63$ \\
\hline Transverse diameter $(\mathrm{cm})$ & $2.48 \pm 0.46$ & $2.47 \pm 0.43$ & $2.36 \pm 0.51$ & $2.05 \pm 0.48$ & $1.94 \pm 0.32$ & $1.86 \pm 0.47$ & $1.95 \pm 0.62$ \\
\hline \multicolumn{8}{|c|}{ 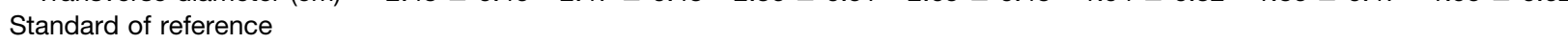 } \\
\hline$n$ & 6 & 6 & 6 & 5 & 5 & 6 & 6 \\
\hline Longitudinal diameter $(\mathrm{cm})$ & $3.86 \pm 0.51$ & $3.93 \pm 0.61$ & $3.56 \pm 0.49$ & $2.91 \pm 0.62$ & $2.31 \pm 0.28$ & $2.34 \pm 0.36$ & $2.0 \pm 0.74$ \\
\hline Transverse diameter $(\mathrm{cm})$ & $2.27 \pm 0.26$ & $2.25 \pm 0.28$ & $2.13 \pm 0.27$ & $1.86 \pm 0.22$ & $1.71 \pm 0.21$ & $1.86 \pm 0.30$ & $1.60 \pm 0.59$ \\
\hline
\end{tabular}

Values are mean \pm SD. $n$ is number of lesions evaluated in each group. Animals that were going on through the longer follow-up period underwent all examinations planned immediately and at the different intervals until planned sacrifice date. Three minipigs were sacrificed after each follow-up imaging procedure. In 2 pigs (1-mo and 2-mo control groups), only 1 radiofrequency ablation could be performed because of hypoplastic left hepatic lobe.

We believe 3 findings from this study to be important. The first of these is that immediately after radiofrequency ablation, local hyperemia caused by outflow obstruction resulted in a peripherally enhancing halo on arterial-phase images, which often was irregular in shape and thickness. The second important finding is that this rimlike area of enhancement could be detected for up to 6 mo after the ablation and might be difficult to differentiate from residual tumor or tumor recurrence on all morphologic imaging modalities. The third is that PET did not show increased uptake on the first postinterventional scan. Therefore, residual tumor seen as a hot spot in the periphery of the ablative necrosis should be detected easily with PET and PET/CT if the tumor is larger than $1 \mathrm{~cm}$. On the basis of these data, ${ }^{18} \mathrm{~F}$-FDG PET or ${ }^{18} \mathrm{~F}-\mathrm{FDG}$ PET/CT performed within the first $2 \mathrm{~h}$ after the intervention may be considered the most suitable imaging modality for therapy control after radiofrequency ablation.

Currently, imaging of patients undergoing interventional liver therapy is based on enhanced CT, enhanced MRI, or enhanced ultrasound (22-25). Although hyperemia is responsible for increased enhancement immediately after the ablation, tissue regeneration in the periphery of the necrotic zone results in a further increase in enhancement during subsequent follow-up. Both phenomena limit the identification of small areas of residual tumor adjacent to the necrotic zone. Failure to detect focal areas of untreated or locally recurrent tumors at an early stage results in tumor enlargement and delayed treatment. Thus, the goal of diagnostic follow-up is to detect residual tumor as soon as possible after radiofrequency ablation.

In the case of unclear enhancing areas in the periphery of the treated tumor, MRI has been proposed as advantageous over spiral CT and ultrasound for monitoring the acute

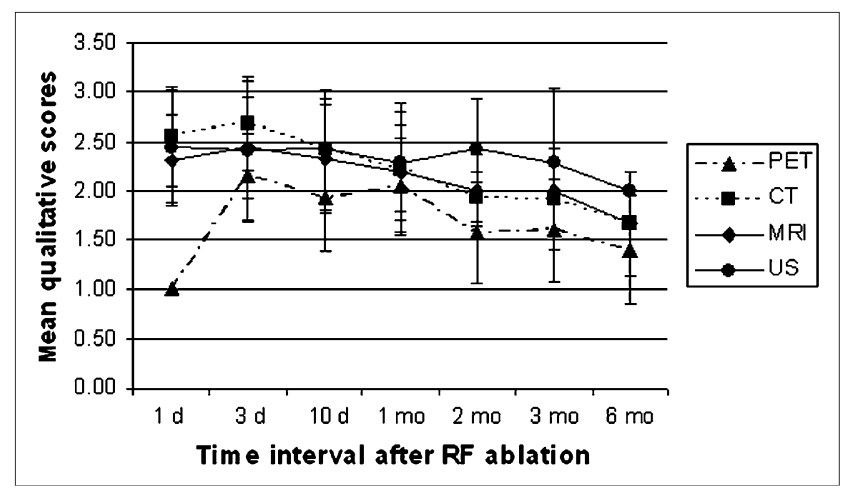

FIGURE 3. Qualitative image analysis according to 3-score scale revealed increased enhancement in periphery of induced necrosis, compared with normal liver parenchyma, on all morphologic imaging modalities. Decrease in intensity can be seen on follow-up-imaging. On PET, no increase in glucose metabolism was found immediately after radiofrequency ablation, leading to score of 1 in all 40 lesions. Peak enhancement surrounding lesions can be seen at $3 \mathrm{~d}$ to 1 mo after radiofrequency ablation. Error bars represent SD. US = ultrasound. 
A

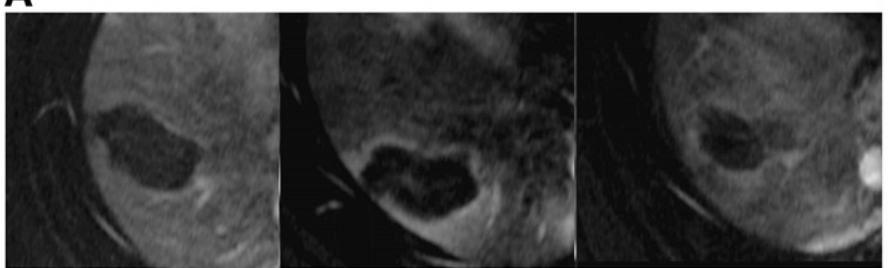

B

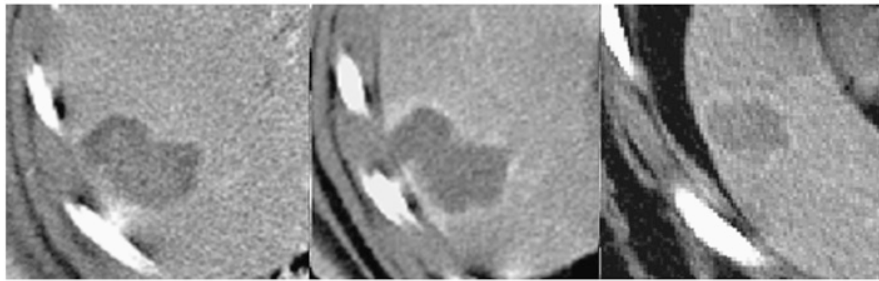

FIGURE 4. Transverse images acquired immediately, $10 \mathrm{~d}$, and 6 mo after radiofrequency ablation demonstrate peripheral enhancement in arterial phase at all follow-up intervals on MRI (A), CT (B), and ultrasound $(\mathrm{C})$. Extent and intensity of enhancement show decay within 6 mo. Functional data provided by PET/CT (1) and PET (2) demonstrate area of decreased ${ }^{18} \mathrm{~F}-\mathrm{FDG}$ uptake surrounded by homogeneous tracer distribution immediately after radiofrequency ablation (D). Tissue regeneration with proliferative activity induced increased glucose metabolism (arrows) from $3 \mathrm{~d}$ (E) to 3 mo after radiofrequency ablation. Six months after interventional procedure, homogeneous tracer distribution can be found again $(F)$.

C

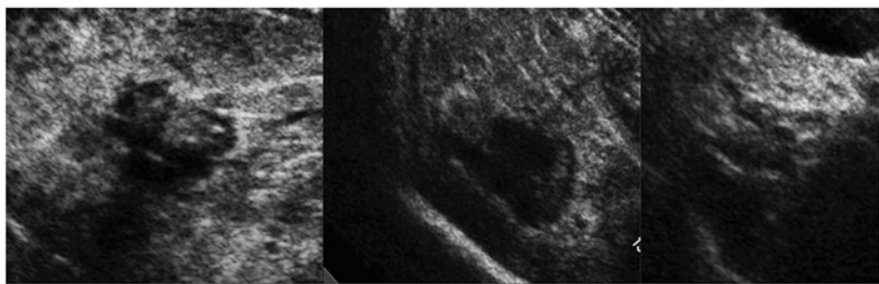

D

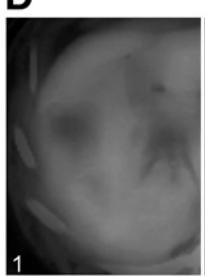

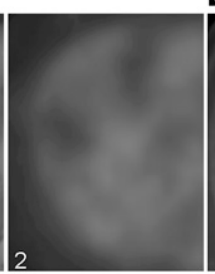

E

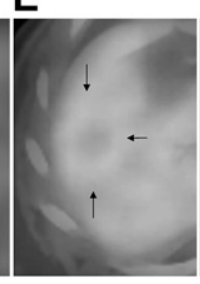

$\mathrm{F}$

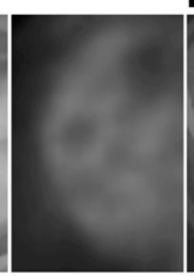

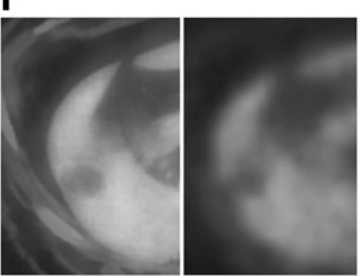

effects of therapy $(23,26,27)$. However, Kim et al. found that dynamic MRI, enhanced using gadopentetate dimeglumine, substantially overestimated the extent of residual tumor after radiofrequency ablation (13). The reason for this overestimation was that enhancement was magnified by the accompanying tissue regeneration, which was described to peak at 1 mo after therapy in that study $(13,27,28)$. The peak enhancement ratios with gadopentetate dimeglumine were almost the same for benign periablational enhancement as for residual tumor. Better differentiation may be obtained with macromolecular (13) or tissue-specific MRI contrast agents such as hepatobiliary and reticuloendothelial system-targeted agents (29-31). Because these agents are generally not taken up by residual viable tumor, it may therefore be distinguished from perfusion abnormalities on late-venous-phase images (32). Using a hepatobiliary MRI contrast agent, we found homogeneous enhancement of the periablational region on late-venous-phase images immediately after and 6 mo after radiofrequency ablation.

Hepatocellular carcinoma and metastases from neuroendocrine tumors typically present as lesions showing early arterial enhancement (33). When radiofrequency ablation is performed on patients with these malignancies, differenti- ation of early arterial hyperemia from residual tumor may be difficult. Although enhancement surrounding the region of radiofrequency ablation was detected over the 6-mo period, a slow decay of the enhancement ratios and a decrease in the mean diameter of the peripheral enhancement were detected over time. One would expect small areas of residual tumor to grow, leading to a further increase in enhancement. Thus, a decrease in enhancement over time will indicate hyperemia and regenerated tissue rather than residual or recurrent tumor. Hence, a close follow-up may reliably detect untreated regions of viable tumor $(1,11)$. Beyond this issue, accurate comparison of pretreatment and posttreatment studies at 3- to 6-mo intervals may further improve diagnostic reliability when differentiating viable tumor from tissue regeneration. However, the objective of this study was not the evaluation of different imaging modalities when differentiating benign from malignant periablational tissue but the assessment of morphologic characteristics after radiofrequency ablation.

When patients with liver metastases from tumors of the gastrointestinal tract are assessed, peripheral enhancement is not expected to cause interpretative problems. On imaging studies, hepatic metastases from gastrointestinal tumors 


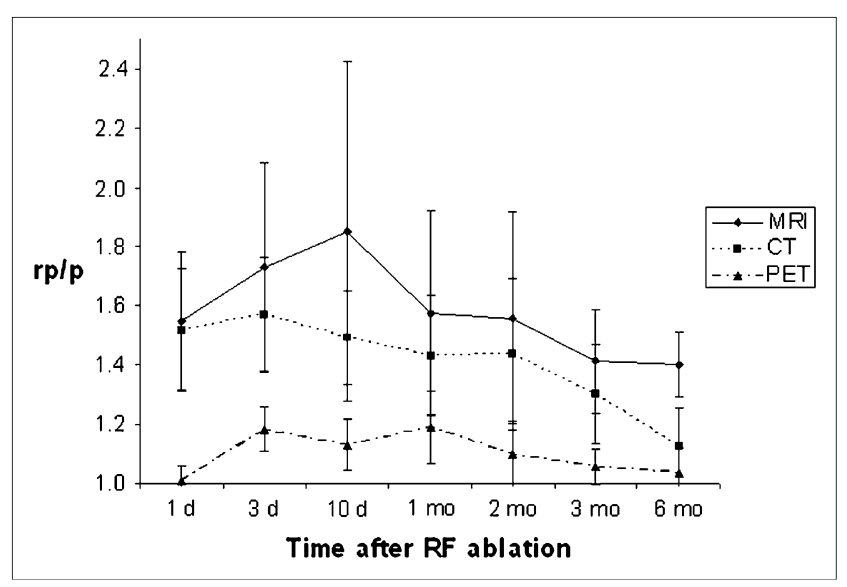

FIGURE 5. Graph shows mean enhancement ratio of lesion periphery to normal liver parenchyma for CT and MRI as well as mean ratio of activity concentration of lesion periphery to activity concentration of normal liver parenchyma on PET. Because of rimlike increase in enhancement at periphery of coagulation, ratios are elevated for morphologic imaging modalities, with slow decay beginning at follow-up interval of $1 \mathrm{mo}$. Homogeneous tracer distribution on PET leads to mean ratio of 1.01 for functional data analysis immediately after radiofrequency ablation, whereas ratio increase of nearly $20 \%$ can be seen between $3 \mathrm{~d}$ and $1 \mathrm{mo}$ after interventional procedure. At 6 mo, interval mean ratio approaches baseline values. Error bars represent SD.

appear mostly as hypodense (CT), hypointense (MRI), or hypoechoic (ultrasound) lesions surrounded by normally enhanced liver parenchyma. In these cases, the peripheral enhancement will not be mistaken for residual tumor, thus not compromising image assessment.

${ }^{18}$ F-FDG PET and ${ }^{18}$ F-FDG PET/CT have been used increasingly not only for the diagnosis, staging, and restaging of malignant tumors but also for therapy assessment after liver interventions. In 3 initial studies, ${ }^{18} \mathrm{~F}-\mathrm{FDG}$ PET and ${ }^{18}$ F-FDG PET/CT were found to be more sensitive than morphologic imaging procedures in detecting residual

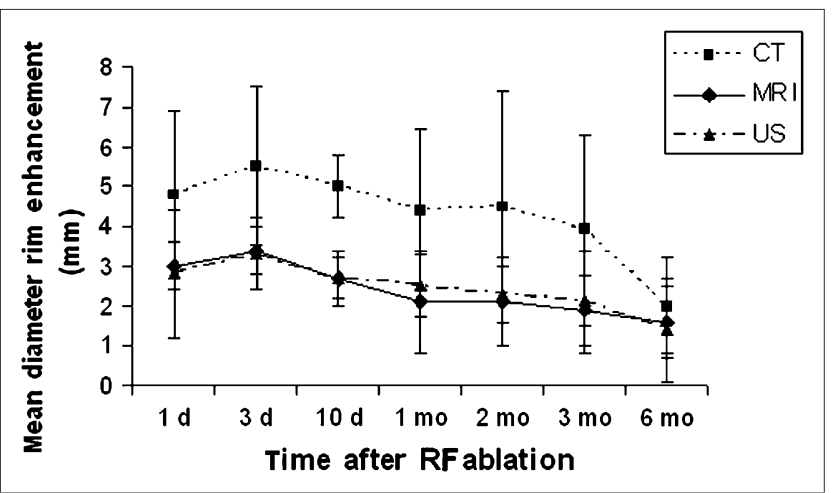

FIGURE 6. Graph shows mean extent of enhancement in periablational region for MRI, CT, and ultrasound (US). Three to $10 \mathrm{~d}$ after radiofrequency ablation, enhancement along ablated tissue became more prominent than immediately after radiofrequency ablation; consequently, slow decay of extent of arterial enhancement can be seen. tumor and tumor recurrence $(14,15,34)$. The time point at which tumor recurrence was detected was considerably earlier for ${ }^{18} \mathrm{~F}-\mathrm{FDG}$ PET than for morphologic imaging (15). However, none of these studies reported the time interval between the liver intervention and PET. This interval must be considered important, because regenerative tissue in the periphery of the ablative necrosis may present as areas of focally increased glucose metabolism (16). Although homogeneous tracer distribution was detected surrounding the area of necrosis immediately after radiofrequency ablation, tissue regeneration with proliferative activity induced increased glucose metabolism from $3 \mathrm{~d}$ to 3 mo after radiofrequency ablation. In our study, both qualitative and quantitative image assessment showed peak tracer uptake between $3 \mathrm{~d}$ and 1 mo after radiofrequency ablation, whereas a decrease to almost baseline values was detected at the 6-mo follow-up. Thus, ${ }^{18}$ F-FDG PET and ${ }^{18} \mathrm{~F}-\mathrm{FDG} \mathrm{PET} / \mathrm{CT}$ for the assessment of radiofrequency ablation should be performed either immediately or $6 \mathrm{mo}$ after radiofrequency ablation.

The additional CT component of combined PET/CT will ensure accurate localization of sites of residual or recurrent tumor. This additional information can be considered crucial for the success of reinterventions to eliminate these areas of tumor. Hence, it seems likely that PET/CT will substantially benefit the early assessment of therapy response to radiofrequency ablation. However, further studies addressing this issue in a clinical setting are required.

Our study had some limitations. The main limitation was the use of normal liver for the radiofrequency ablation procedure. Cirrhotic liver and liver tumors may conduct the radiofrequency current differently and may show a different extent of regenerative tissue in the periphery of the necrosis. Thus, imaging characteristics may vary. Further studies are required to address the question of the extent to which tissue regeneration obscures residual or recurrent tumor. Up to now, there has been no model of liver tumors or metastases in pigs, and our own series designed to implement a rodent tumor cell line in domestic pigs showed no useful results. Even if rodent models for percutaneous tumor ablation of liver lesions were to exist, these models would not be suitable for long-term follow-up intervals of up to 6 mo.

Because this study examined only healthy animals, further research is needed to evaluate whether the potential advantage of PET and PET/CT over morphologic imaging immediately after radiofrequency ablation translates into a faster and more accurate assessment of residual tumor in patients who survive longer after ablation.

The comparison of morphologic imaging and ${ }^{18} \mathrm{~F}-\mathrm{FDG}$ PET or ${ }^{18} \mathrm{~F}-\mathrm{FDG}$ PET/CT is useful only for ${ }^{18} \mathrm{~F}$-FDG PETpositive tumors. Hepatocellular carcinoma and neuroendocrine tumors often show no increased glucose metabolism. In these cases, more specific tracers such as ${ }^{68} \mathrm{Ga}$-DOTATOC for neuroendocrine carcinoma should be used when comparing morphologic and functional imaging findings. 


\section{CONCLUSION}

Detection of residual tumor with ${ }^{18} \mathrm{~F}$-FDG PET and ${ }^{18} \mathrm{~F}$ FDG PET/CT immediately or 6 mo after radiofrequency ablation of liver lesions will not be hampered by increased cell metabolism. However, between these 2 follow-up dates, increased tracer uptake due to regenerative tissue may hamper the detection of residual tumor. In contrast, morphologic imaging findings may be difficult to interpret immediately and up to 6 mo after radiofrequency ablation because of increased periablational enhancement.

\section{ACKNOWLEDGMENTS}

We thank Sandra Massing, Michaela Jökel, and Silke Bosk for conducting the MRI examinations. We gratefully acknowledge the substantial support of Dirk Lahmer in providing ${ }^{18} \mathrm{~F}$-FDG for the animal experiments. This work was supported by a grant from the Internal Foundation of Research, Essen (IFORES).

\section{REFERENCES}

1. Solbiati L, Livraghi T, Goldberg $\mathrm{SN}$, et al. Percutaneous radio-frequency ablation of hepatic metastases from colorectal cancer: long-term results in 117 patients. Radiology. 2001;221:159-166.

2. McGhana JP, Dodd GD III. Radiofrequency ablation of the liver: current status. AJR. 2001;176:3-16.

3. Solbiati L, Goldberg SN, Ierace T, et al. Hepatic metastases: percutaneous radiofrequency ablation with cooled-tip electrodes. Radiology. 1997;205:367-373.

4. Rossi S, Buscarini E, Garbagnati F, et al. Percutaneous treatment of small hepatic tumors by an expandable RF needle electrode. AJR. 1998;170:1015-1022.

5. Dupuy DE, Goldberg SN. Image-guided radiofrequency tumor ablation: challenges and opportunities-part II. J Vasc Interv Radiol. 2001;12:1135-1148.

6. Kuvshinoff BW, Ota DM. Radiofrequency ablation of liver tumors: influence of technique and tumor size. Surgery. 2002;132:605-611.

7. Wood TF, Rose DM, Chung M, Allegra DP, Foshag LJ, Bilchik AJ. Radiofrequency ablation of 231 unresectable hepatic tumors: indications, limitations, and complications. Ann Surg Oncol. 2000;7:593-600.

8. Solbiati L, Ierace T, Tonolini M, Osti V, Cova L. Radiofrequency thermal ablation of hepatic metastases. Eur J Ultrasound. 2001;13:149-158.

9. Ng KK, Lam CM, Poon RT, et al. Porcine liver: morphologic characteristics and cell viability at experimental radiofrequency ablation with internally cooled electrodes. Radiology. 2005;235:478-486.

10. Goldberg SN, Gazelle GS, Compton CC, Mueller PR, Tanabe KK. Treatment of intrahepatic malignancy with radiofrequency ablation: radiologic-pathologic correlation. Cancer. 2000;88:2452-2463.

11. Goldberg SN, Charboneau JW, Dodd GD III, et al. Image-guided tumor ablation: proposal for standardization of terms and reporting criteria. Radiology. 2003; 228:335-345.

12. Choi H, Loyer EM, DuBrow RA, et al. Radio-frequency ablation of liver tumors: assessment of therapeutic response and complications. Radiographics. 2001;21: S41-S54.
13. Kim TJ, Moon WK, Cha JH, et al. VX2 carcinoma in rabbits after radiofrequency ablation: comparison of MR contrast agents for help in differentiating benign periablational enhancement from residual tumor. Radiology. 2005;234: 423-430.

14. Anderson GS, Brinkmann F, Soulen MC, Alavi A, Zhuang H. FDG positron emission tomography in the surveillance of hepatic tumors treated with radiofrequency ablation. Clin Nucl Med. 2003;28:192-197.

15. Langenhoff BS, Oyen WJ, Jager GJ, et al. Efficacy of fluorine-18-deoxyglucose positron emission tomography in detecting tumor recurrence after local ablative therapy for liver metastases: a prospective study. J Clin Oncol. 2002;20:44534458.

16. Antoch G, Freudenberg LS, Beyer T, Bockisch A, Debatin JF. To enhance or not to enhance? ${ }^{18} \mathrm{~F}$-FDG and CT contrast agents in dual-modality ${ }^{18} \mathrm{~F}-\mathrm{FDG}$ PET/CT. J Nucl Med. 2004;45(suppl 1):56S-65S.

17. Kruskal JB, Oliver B, Huertas JC, Goldberg SN. Dynamic intrahepatic flow and cellular alterations during radiofrequency ablation of liver tissue in mice. $J$ Vasc Interv Radiol. 2001;12:1193-1201.

18. Lazebnik RS, Breen MS, Fitzmaurice M, Nour SG, Lewin JS, Wilson DL. Radio-frequency-induced thermal lesions: subacute magnetic resonance appearance and histological correlation. J Magn Reson Imaging. 2003;18:487-495.

19. Antoch G, Vogt FM, Veit P, et al. Assessment of liver tissue after radiofrequency ablation: findings with different imaging procedures. J Nucl Med. 2005;46:520-525.

20. Lencioni R, Goletti O, Armillotta N, et al. Radio-frequency thermal ablation of liver metastases with a cooled-tip electrode needle: results of a pilot clinical trial. Eur Radiol. 1998;8:1205-1211.

21. Kinahan PE, Townsend DW, Beyer T, Sashin D. Attenuation correction for a combined 3D PET/CT scanner. Med Phys. 1998;25:2046-2053.

22. Cioni D, Lencioni R, Bartolozzi C. Percutaneous ablation of liver malignancies: imaging evaluation of treatment response. Eur J Ultrasound. 2001;13:73-93.

23. Dromain C, de Baere T, Elias D, et al. Hepatic tumors treated with percutaneous radio-frequency ablation: CT and MR imaging follow-up. Radiology. 2002; 223:255-262.

24. Tacke J. Percutaneous radiofrequency ablation: clinical indications and results [in German]. RöFo. 2003;175:156-168.

25. Lencioni R, Cioni D, Crocetti L, et al. Early-stage hepatocellular carcinoma in patients with cirrhosis: long-term results of percutaneous image-guided radiofrequency ablation. Radiology. 2005;234:961-967.

26. Yan FH, Shen JZ, Li RC, et al. Enhancement patterns of small hepatocellular carcinoma shown by dynamic MRI and CT. Hepatobiliary Pancreat Dis Int. 2002; $1: 420-424$.

27. Bipat S, van Leeuwen MS, Comans EF, et al. Colorectal liver metastases: CT, MR imaging, and PET for diagnosis-meta-analysis. Radiology. 2005;237:123-131.

28. Sironi S, Livraghi T, Meloni F, De Cobelli F, Ferrero C, Del Maschio A. Small hepatocellular carcinoma treated with percutaneous RF ablation: MR imaging follow-up. AJR. 1999;173:1225-1229.

29. Tanimoto A, Kuwatsuru R, Kadoya M, et al. Evaluation of gadobenate dimeglumine in hepatocellular carcinoma: results from phase II and phase III clinical trials in Japan. J Magn Reson Imaging. 1999;10:450-460.

30. Harisinghani MG, Jhaveri KS, Weissleder R, et al. MRI contrast agents for evaluating focal hepatic lesions. Clin Radiol. 2001;56:714-725.

31. Bluemke DA, Sahani D, Amendola M, et al. Efficacy and safety of MR imaging with liver-specific contrast agent: U.S. multicenter phase III study. Radiology. 2005;237:89-98.

32. Puls R, Kroncke TJ, Kluner C, et al. Double contrast MRI of thermally ablated liver metastases [in German]. RöFo. 2003;175:1467-1470.

33. Paul SB, Gulati MS. Spectrum of hepatocellular carcinoma on triple phase helical CT: a pictorial essay. Clin Imaging. 2002;26:270-279.

34. Veit P, Antoch G, Stergar H, Bockisch A, Forsting M, Kuehl H. Detection of residual tumor after radiofrequency ablation of liver metastasis with dualmodality PET/CT: initial results. Eur Radiol. 2006;16:80-87. 